\title{
Tratamento de sequelas do Acidente Vascular Encefálico (AVE) com toxina
}

\section{botulínica: Uma revisão de literatura}

\author{
Treatment of Cerebrovascular Accident (CVA) sequelae with botulinum toxin: A literature review \\ Tratamiento de las secuelas del Accidente Cerebrovascular (ACV) con toxina botulínica: Revisión \\ de la literatura
}

Recebido: 05/07/2021 | Revisado: 08/07/2021 | Aceito: 09/07/2021 | Publicado: 18/07/2021

\author{
Bárbara Queiroz de Figueiredo \\ ORCID: https://orcid.org/0000-0003-1630-4597 \\ Centro Universitário de Patos de Minas, Brasil \\ E-mail: barbarafigueiredo@unipam.edu.br \\ Antônio Ricardo Neto \\ ORCID: https://orcid.org/0000-0001-6647-9374 \\ Centro Universitário de Patos de Minas, Brasil \\ E-mail:antonioneto12@unipam.edu.br \\ Caio Flávio Reis Nogueira \\ ORCID: https://orcid.org/0000-0003-1514-9660 \\ Centro Universitário de Patos de Minas, Brasil \\ E-mail: caioflavio@unipam.edu.br \\ Rafaela Alves Fernandes \\ ORCID: https://orcid.org/0000-0002-0050-0844 \\ Centro Universitário de Patos de Minas, Brasil \\ E-mail: rafaelaaf@unipam.edu.br \\ Marcelo Gomes de Almeida \\ ORCID: https://orcid.org/0000-0002-6297-4383 \\ Universidade Federal de Minas Gerais, Brasil \\ E-mail: marcelomedile@yahoo.com.br
}

\begin{abstract}
Resumo
Introdução: O acidente vascular encefálico (AVE) é um evento bastante prevalente na população mundial, e dentre suas sequelas, destaca-se a espasticidade muscular e paralisias. A toxina botulínica é uma técnica farmacológica que traz efeitos benéficos ao paciente com espasticidade, como o ganho de amplitude de movimento e melhora da função dos membros afetados. Objetivo: identificar as evidências acerca do uso dessa toxina a esses pacientes, principalmente perante a espasticidade muscular, evento fortemente relacionado após AVE. Metodologia: Trata-se de uma pesquisa descritiva do tipo revisão integrativa da literatura, que busca evidências sobre o uso da toxina botulínica para tratamento de sequelas do AVE. A pesquisa foi realizada por meio de bases de dados PubMed, MEDLINE, Scielo, CDSR, Google Scholar, BVS e EBSCO, no período de 2003 a 2021. Dessa maneira, totalizaram-se 26 produções científicas para a revisão integrativa da literatura. Resultados: o tratamento da espasticidade com a toxina botulínica tem maior êxito quando a administração segue alguns critérios como: o ajuste da dose de acordo com a idade, com o peso, grau de espasticidade e musculatura administrada. Conclusão: a aplicação dessa toxina, aliado à fisioterapia continuada, é o tratamento de primeira escolha para a espasticidade muscular, particularmente em pacientes que apresentam sequelas pós acidente vascular cerebral, com o intuito de aumentar a mobilidade, amplitude de movimento, facilitar a realização da higiene e de outras atividades funcionais, melhorar o desgaste da imobilização e a dor, e, dessa maneira, promover a melhoria da qualidade de vida desses pacientes.
\end{abstract}

Palavras-chave: Toxina botulínica; Paralisia; Acidente vascular cerebral; Reabilitação; Espasticidade muscular.

\begin{abstract}
Introduction: The cerebrovascular accident (CVA) is a very prevalent event in the world population, and among its sequelae, muscle spasticity and paralysis stand out. Botulinum toxin is a pharmacological technique that brings beneficial effects to patients with spasticity, such as gain in range of motion and improvement in the function of affected limbs. Objective: to identify the evidence regarding the use of this toxin in these patients, especially in the face of muscle spasticity, an event strongly related to a stroke. Methodology: This is a descriptive research of the integrative literature review type, which seeks evidence on the use of botulinum toxin for the treatment of stroke sequelae. The search was conducted using PubMed, MEDLINE, Scielo, CDSR, Google Scholar, BVS and EBSCO databases, from 2003 to 2021. Thus, 26 scientific productions were totaled for the integrative literature review. Results: the treatment of spasticity with botulinum toxin is more successful when the administration follows some criteria such as: dose adjustment according to age, weight, degree of spasticity and musculature administered.
\end{abstract}


Conclusion: the application of this toxin, combined with continued physical therapy, is the treatment of first choice for muscle spasticity, particularly in patients with sequelae after stroke, in order to increase mobility, range of motion, facilitate the performance of hygiene and other functional activities, improve immobilization wear and pain, and, thus, improve the quality of life of these patients.

Keywords: Botulinum toxin; Paralysis; Stroke; Rehabilitation; Muscle spasticity.

\begin{abstract}
Resumen
Introducción: El accidente cerebrovascular (ACV) es un evento muy prevalente en la población mundial, y entre sus secuelas destacan la espasticidad muscular y la parálisis. La toxina botulínica es una técnica farmacológica que aporta efectos beneficiosos a los pacientes con espasticidad, como la ganancia en el rango de movimiento y la mejora de la función de las extremidades afectadas. Objetivo: identificar la evidencia sobre el uso de esta toxina en estos pacientes, especialmente ante la espasticidad muscular, evento fuertemente relacionado con un ictus. Metodología: Se trata de una investigación descriptiva del tipo revisión integradora de la literatura, que busca evidencia sobre el uso de la toxina botulínica para el tratamiento de las secuelas del ictus. La búsqueda se realizó utilizando las bases de datos PubMed, MEDLINE, Scielo, CDSR, Google Scholar, BVS y EBSCO, de 2003 a 2021. Así, se totalizaron 26 producciones científicas para la revisión integradora de la literatura. Resultados: el tratamiento de la espasticidad con toxina botulínica es más exitoso cuando la administración sigue algunos criterios como: ajuste de dosis según edad, peso, grado de espasticidad y musculatura administrada. Conclusión: la aplicación de esta toxina, combinada con fisioterapia continuada, es el tratamiento de primera elección para la espasticidad muscular, particularmente en pacientes con secuelas tras un ictus, con el fin de incrementar la movilidad, amplitud de movimiento, facilitar la realización de la higiene y otros aspectos funcionales. actividades, mejoran el desgaste y el dolor de la inmovilización y, por tanto, mejoran la calidad de vida de estos pacientes.
\end{abstract}

Palabras clave: Toxina botulínica; Parálisis; Accidente vascular cerebral; Rehabilitación; Espasticidad muscular.

\title{
1. Introdução
}

$\mathrm{O}$ crescente envelhecimento populacional tem elevado o número de pessoas com doenças e incapacidades crônicas, dentre elas, o Acidente Vascular Cerebral (AVC), também chamado de Acidente Vascular Encefálico (AVE), sendo uma das grandes preocupações da atualidade, o que gera repercussões sociais na saúde pública e na previdência social, fator que tem grande impacto econômico e social. Aliado a isso, o termo "Doenças Cerebrovasculares (DCV)" é utilizado para se referir a anormalidades do encéfalo decorrentes de alterações do seu sistema vascular, que se originam de diversos mecanismos fisiopatológicos, como tromboses, embolias, ruptura de vasos, alterações da permeabilidade vascular, aumento da viscosidade sanguínea ou alterações qualitativas do suprimento sanguíneo (Schuster et al., 2007).

A Organização Mundial de Saúde define o Acidente Vascular Encefálico (AVE) como um distúrbio focal da função cerebral, desenvolvido rapidamente, perdurando por mais de 24 horas, sendo de origem vascular. O AVE, classificado como uma doença cerebrovascular, no mundo, é considerada a terceira causa de morte, sendo superado apenas por neoplasias e doenças cardiovasculares (Calil et al., 2007). Estudos sobre a prevalência desta enfermidade calculam que há cerca de 5 a 10 casos de AVE a cada 1000 habitantes, em todo o mundo, sendo que a doença atinge em torno de 17 milhões de pessoas por ano em todo mundo, aproximadamente $60 \%$ dos sobreviventes recupere a independência para o autocuidado, $75 \%$ recupere a marcha independente e 20\% exigirão cuidados institucionais. De acordo com Almeida (2012) no Brasil, o AVE é uma das principais causas de internações e mortalidade, causando na grande maioria dos pacientes, algum tipo de deficiência, seja parcial ou completa.

O AVE pode ser de origem isquêmica (obstrução vascular localizada, que leva a interrupção do fornecimento de oxigênio e glicose ao cérebro, afetando subsequentemente os processos metabólicos do território envolvido) ou hemorrágico (causado por um aneurisma ou trauma dentro das áreas extravasculares do cérebro) (Piassaroli et al., 2012). Independentemente do tipo (isquêmico ou hemorrágico), o AVE causa limitações funcionais diversas pela perda da autonomia decorrente de incapacidades geradas, principalmente no tocante à espasticidade, que é um comum causado pela lesão do sistema nervoso central, caracterizada por aumento da resistência ao alongamento muscular passivo, dependente da velocidade de estiramento do músculo, ocasionando hiperexcitabilidade dos reflexos miotáticos, hipertonia elástica, alteração da 
sensibilidade proprioceptiva, sendo, muitas vezes acompanhados por clônus, espasmos flexor e/ou extensor, contraturas, hiperreflexia autonômica, distonia e por reflexos patológicos como Babinski (Teles et al., 2012).

De acordo com Thompson et al. (2005), a espasticidade é uma síndrome que dificulta a reabilitação neuromotora dos pacientes com AVE, podendo diminuir a amplitude de movimento articular, causar dores, limitar as atividades dos membros, como também pode dificultar as atividades funcionais e de vida prática, como, por exemplo, a higiene pessoal e os cuidados com a aparência exterior, sendo responsável também por causar alterações na marcha e como um dos fatores que interferem no processo de reabilitação funcional após Acidente Vascular Encefálico. Ela surge por causa da perda da inibição do reflexo miotático, resultante de lesão do neurônio motor superior (Pierson et al., 1996). Os pacientes com espasticidade são frequentemente hemiparéticos, acometendo membro superior e membro inferior, dependendo do lado cerebral acometido, possuindo uma maior incapacidade dos que os pacientes sem espasticidade (Lundstrom et al., 2008).

Dessa forma, a espasticidade é uma sequela motora muito incapacitante, presente na maior parte dos casos de AVE, limitando as atividades funcionais, o que se faz necessária a avaliação e intervenção sobre a mesma, tendo em vista que o aumento do tônus muscular gera grande incapacidade funcional interferindo na qualidade de vida desta população de pacientes. Para mensuração do grau de espasticidade, usualmente e clinicamente é utilizada uma escala específica, chamada, "Escala Modificada de Ashworth", que é realizada por meio da mobilização passiva da extremidade através do arco de movimento, para afastar origem de inserção de grupos musculares, quantificando a resistência ao movimento de forma rápida nas diversas articulações (Teles et al., 2012).

Sob esse cenário, o tratamento através da toxina botulínica é considerado um padrão-ouro para reabilitação de pacientes com sequelas pós AVE, haja vista que age através do bloqueio químico da transmissão neuromuscular via inibição da liberação de acetilcolina. É um medicamento advindo do extrato purificado formado por um complexo proteico de origem biológica, obtido a partir do Clostridium Botulinum, uma bactéria anaeróbia que, em condições apropriadas à sua reprodução $\left(10^{\circ} \mathrm{C}\right.$, sem oxigênio e certo nível de acidez), cresce e produz sete sorotipos diferentes ou sete cadeias imunologicamente distintas de neurotoxinas: A, B, C, D, E, F e G. Dentre esses, o sorotipo A é o mais potente e o que proporciona maior duração de efeitos, utilizado tanto para fins terapêuticos como estéticos, haja vista que seus efeitos clínicos têm sido reconhecidos desde o final do século XIX, caracterizado por ser um medicamento produzido por biossíntese em células vivas, diferente dos sintéticos que são obtidos por síntese química (Mukai et al., 2011).

Diante dessas evidências, o tratamento de indivíduos com sequelas de AVE com o uso da toxina botulínica na modulação da espasticidade tem tido grande aceitação pelos pacientes, pois este tratamento tem ocasionado ganho na Amplitude de Movimento (ADM), melhora capacidade funcional e qualidade de vida e acredita-se que pacientes com sequelas de AVE apresentam melhora da qualidade de vida e capacidade funcional após aplicação da toxina botulínica tipo A (Gouvêa et al., 2015). As pesquisas acerca do assunto têm feito com que sejam desenvolvidas evidências e expostas experiências da aplicação de toxina botulínica A em uma série de pacientes com sequelas pós AVE, e, desse modo, o presente estudo visa identificar as evidências acerca do uso dessa toxina a esses pacientes, principalmente perante a espasticidade muscular, evento fortemente relacionado após AVE.

\section{Metodologia}

Trata-se de uma pesquisa descritiva do tipo revisão integrativa da literatura, que buscou responder quais as evidências sobre o uso da toxina botulínica no tratamento de sequelas pós acidente vascular cerebral (AVE). A pesquisa foi realizada através do acesso online nas bases de dados National Library of Medicine (PubMed MEDLINE), Scientific Electronic Library Online (Scielo), Cochrane Database of Systematic Reviews (CDSR), Google Scholar, Biblioteca Virtual em Saúde (BVS) e EBSCO Information Services, no mês de julho de 2021. 
Para a busca das obras foram utilizadas as palavras-chaves presentes nos descritores em Ciências da Saúde (DeCS): em inglês: "botulinum toxi", "paralysis", "stroke", "rehabilitation", "muscle spasticity", em português: "toxina botulinica", "paralisia", "acidente vascular cerebral", "reabilitação", "espasticidade muscular", em espanhol: "toxina botulínica", "parálisis", "accidente vascular cerebral", "rehabilitación", "espasticidad muscular" e em francês: "toxine botulique" "paralysie”, “coup", “réhabilitation”, "spasticité musculaire”.

Como critérios de inclusão, foram considerados artigos originais, que abordassem o tema pesquisado e permitissem acesso integral ao conteúdo do estudo, publicados no período de 2003 a 2021, em inglês, português, espanhol e francês. O critério de exclusão foi imposto naqueles trabalhos que não estavam em inglês, português, espanhol e francês que não tinham passado por processo de Peer-View e que não relacionassem a utilização da toxina botulínica para tratamento de sequelas pós AVE.

A estratégia de seleção dos artigos seguiu as seguintes etapas: busca nas bases de dados selecionadas; leitura dos títulos de todos os artigos encontrados e exclusão daqueles que não abordavam o assunto; leitura crítica dos resumos dos artigos e leitura na íntegra dos artigos selecionados nas etapas anteriores. Após leitura criteriosa das publicações, 6 artigos não foram utilizados devido aos critérios de exclusão. Assim, totalizaram-se 26 artigos científicos para a revisão integrativa da literatura, com os descritores apresentados acima. Após esta seleção, filtraram-se por artigos dos últimos dezoito anos e por artigos em línguas portuguesa, inglesa, espanhola e francesa.

\section{Resultados e Discussão}

Após a seleção dos artigos por meio dos critérios de inclusão e de exclusão, elaborou-se uma tabela (Tabela 1) contendo as principais informações sobre o uso da toxina botulínica em pacientes com sequelas pós-AVE.

Tabela 1: Estudos utilizados na Revisão Integrativa e achados principais.

\begin{tabular}{|c|c|c|}
\hline AUTOR & ANO & PRINCIPAIS ACHADOS \\
\hline $\begin{array}{l}\text { Gouvêa, D., et } \\
\text { al. }\end{array}$ & 2015 & $\begin{array}{l}\text { O tratamento de indivíduos com sequelas de AVE com o uso da toxina botulínica na modulação da } \\
\text { espasticidade tem tido grande aceitação pelos pacientes, pois este tratamento tem ocasionado ganho na } \\
\text { amplitude de movimento (ADM), melhora capacidade funcional e qualidade de vida. }\end{array}$ \\
\hline $\begin{array}{l}\text { Sposito, M. M. } \\
\text { M. }\end{array}$ & 2009 & $\begin{array}{l}\text { A Toxina Botulínica tipo A é uma terapia farmacológica que traz efeitos benéficos ao paciente com } \\
\text { espasticidade, como o ganho de amplitude de movimento e melhora da função dos membros afetados. }\end{array}$ \\
\hline $\begin{array}{l}\text { Souza, O. A., et } \\
\text { al. }\end{array}$ & 2016 & $\begin{array}{l}\text { O uso da toxina botulínica facilita o controle das contrações. No tratamento da espasticidade, a utilização da } \\
\text { toxina botulínica tipo A, em conjunto com Fisioterapia, é um método útil para reabilitação de pacientes que } \\
\text { apresentam espasticidade, como os que tiveram sequelas pós AVE, e inclusive, para os que tiveram } \\
\text { Traumatismo Crânio Encefálico (TCE) }\end{array}$ \\
\hline $\begin{array}{l}\text { Portella, L. V., } \\
\text { et al. }\end{array}$ & 2004 & $\begin{array}{l}\text { A toxina botulínica atua bloqueando a liberação de acetilcolina no terminal pré-sináptico através da } \\
\text { desativação das proteínas de fusão, impedindo que a acetilcolina seja lançada na fenda sináptica e, dessa } \\
\text { maneira, não permitindo a despolarização do terminal pós-sináptico e bloqueando, assim, a contração } \\
\text { muscular. Este medicamento, entretanto, não interfere na produção da acetilcolina e, por este motivo, o } \\
\text { bloqueio é reversível alguns meses após a aplicação. }\end{array}$ \\
\hline $\begin{array}{l}\text { Sposito, M. M. } \\
\text { M., et al. }\end{array}$ & 2004 & $\begin{array}{l}\text { Quanto ao mecanismo de ação, a toxina botulínica tipo A age sobre a inibição da liberação de acetilcolina } \\
\text { nos terminais nervosos motores e na ação sobre outros neurotransmissores, promovendo relaxamento } \\
\text { muscular (ação sobre músculos estriados; ação sobre o reflexo de estiramento medular), sendo de grande } \\
\text { valia no tratamento para pacientes com sequelas pós AVE. }\end{array}$ \\
\hline Foley, N., et al. & 2013 & $\begin{array}{l}\text { A administração deste fármaco está limitada a músculos específicos em doses controladas. Recomenda-se a } \\
\text { injeção intramuscular em cada músculo comprometido com a espasticidade, injeções subcutâneas podem } \\
\text { ser indicadas em situações especiais. }\end{array}$ \\
\hline Fujimura, O. T. & 2017 & Ao utilizar a escala de avaliação de incapacidade, os escores para higiene e curativo melhoraram \\
\hline
\end{tabular}




\begin{tabular}{|c|c|c|}
\hline R. K. & & $\begin{array}{l}\text { significativamente } 2 \text { e } 6 \text { semanas após a administração da toxina botulínica, mas não houve mudança } \\
\text { significativa na dor, e os escores totais para parâmetros de membros superiores exibiram melhora } \\
\text { significativa entre } 2 \text { e } 12 \text { semanas após sua administração. }\end{array}$ \\
\hline $\begin{array}{l}\text { Barbosa, C. M. } \\
\text { R. }\end{array}$ & 2017 & $\begin{array}{l}\text { As associações realizadas entre as variáveis evidenciaram a existência de chances maiores para ocorrência } \\
\text { do desfecho favorável em pacientes com paralisia facial após uso da toxina botulínica tipo A, em relação à } \\
\text { espasticidade e funcionalidade, quando comparado aos indivíduos que não receberam essa intervenção. }\end{array}$ \\
\hline $\begin{array}{l}\text { Lima, L. S. R., } \\
\text { et al. }\end{array}$ & 2020 & $\begin{array}{l}\text { Seu efeito é reversível, durando aproximadamente de } 3 \text { a } 6 \text { meses, sendo esse período dependente do } \\
\text { paciente, da frequência e quantidade de dose aplicada, ou seja, a aplicação da toxina botulínica consiste em } \\
\text { um tratamento reversível de curto prazo. }\end{array}$ \\
\hline $\begin{array}{l}\text { Anjos, A. A. S., } \\
\text { et al. }\end{array}$ & 2020 & $\begin{array}{l}\text { Relato de caso clínico mostrou efeitos positivos em relação ao tratamento com Toxina Botulínica A para } \\
\text { correção de assimetria facial por paralisia pós AVE, foi eficaz para devolver estética, simetria, função e } \\
\text { equilíbrio muscular à face da paciente, sendo possível observar a satisfação da mesma ao final do } \\
\text { tratamento. }\end{array}$ \\
\hline $\begin{array}{l}\text { Ênia, J. R. N., et } \\
\text { al. }\end{array}$ & 2021 & $\begin{array}{l}\text { O uso da toxina botulínica na reabilitação de pacientes com paralisia facial vem aumentando cada vez mais } \\
\text { e os estudos a respeito do produto estão em crescimento, de forma a se alcançar um protocolo único de } \\
\text { utilização, mesmo que ainda pode-se notar escassez desses estudos. A reabilitação dos pacientes com } \\
\text { paralisia facial utilizando a toxina botulínica é eficaz e segura, sendo uma opção, principalmente, para } \\
\text { melhorar a assimetria facial. }\end{array}$ \\
\hline $\begin{array}{l}\text { Pimentel, L. H. } \\
\text { C., et al. }\end{array}$ & 2014 & $\begin{array}{l}\text { Em pacientes com hemiparesia espástica secundária ao AVE, à aplicação de TBA com tempo de intervalo } \\
\text { do evento superior a } 6 \text { meses, os resultados demonstraram melhoria da espasticidade. No entanto, A } \\
\text { melhora da velocidade de marcha e da independência funcional não foram correlacionadas com a dose de } \\
\text { TBA na amostra analisada. }\end{array}$ \\
\hline $\begin{array}{l}\text { Figallo, S. M. } \\
\text { A., et al. }\end{array}$ & 2020 & $\begin{array}{l}\text { A neurotoxina botulínica é um produto altamente eficaz e potente que pode ser usado de forma terapêutica, } \\
\text { e dentro da odontologia ela tem sido utilizada em diversos tratamentos, como no equilíbrio facial em } \\
\text { pacientes que apresentam paralisia facial. Com isso os pacientes passam a ter um condicionamento da } \\
\text { atividade muscular, promovendo um equilíbrio na simetria da face, através de um tratamento menos } \\
\text { invasivo que melhora além de questões funcionais, a autoestima e questões psicológicas do indivíduo. }\end{array}$ \\
\hline $\begin{array}{l}\text { Oliveira, D. R. } \\
\text { N., et al. }\end{array}$ & 2017 & $\begin{array}{l}\text { Os efeitos benéficos do uso da toxina botulínica no tratamento da espasticidade são evidenciados na Escala } \\
\text { de Ashworth, e parecem potencializar o tratamento fisioterapêutico. No entanto, algumas contraindicações } \\
\text { podem ser citadas, como: doenças neuromusculares, hipersensibilidade à toxina botulínica, gestantes e uso } \\
\text { antibióticos. }\end{array}$ \\
\hline Casaca, I. & 2006 & $\begin{array}{l}\text { Cerca de } 10 \% \text { dos pacientes tratados com TBA desenvolvem anticorpos à toxina, e segundo pesquisas, isso } \\
\text { pode acontecer por administração de doses altas de TB em pequenos intervalos de tempo. Por isso, } \\
\text { recomenda-se que as doses administradas sejam as menores possíveis por sessão. }\end{array}$ \\
\hline Cardoso, E. & 2003 & $\begin{array}{l}\text { O uso da TBA em } 20 \text { pacientes com espasticidade após AVE foi capaz de melhorar a amplitude de } \\
\text { movimento e o score na Escala de Ashworth. Neste estudo, os pacientes apresentaram melhoras também na } \\
\text { escala de Medida da Independência Funcional (MIF). }\end{array}$ \\
\hline $\begin{array}{l}\text { Ward, A. B, et } \\
\text { al. }\end{array}$ & 2014 & $\begin{array}{l}\text { Em pacientes com hemiplegia e hemiparesia espástica secundária ao AVE, a aplicação da TBA, com tempo } \\
\text { de intervalo do evento igual ou superior a } 3 \text { meses, resultou em uma menor espasticidade, maior } \\
\text { deambulação e melhoria nas atividades funcionais de vida diária. }\end{array}$ \\
\hline $\begin{array}{l}\text { Wolfgang, H. J, } \\
\text { et al. }\end{array}$ & 2014 & $\begin{array}{l}\text { Em pacientes com sequelas secundárias ao AVE, a aplicação de TBA, com tempo médio de intervalo do } \\
\text { evento entre } 7 \text { meses, com quantidade e doses dependentes do protocolo e do aplicador, os resultados foram } \\
\text { menor espasticidade e melhora no quadro de dor. }\end{array}$ \\
\hline $\begin{array}{l}\text { Veverka, T., et } \\
\text { al. }\end{array}$ & 2016 & $\begin{array}{l}\text { Os resultados da aplicação de TBA em pacientes com sequelas pós AVE foram a diminuição da } \\
\text { espasticidade, aumento da atividade funcional, da deambulação, da atividade cortical, do controle motor e } \\
\text { da potência muscular na região tratada. }\end{array}$ \\
\hline Serrano, S, et al. & 2014 & $\begin{array}{l}\text { O tratamento com TBA é eficaz na redução da espasticidade e na melhoria das amplitudes articulares dos } \\
\text { membros superiores. A escala Goal Attainment Scaling (GAS) mostrou-se uma ferramenta sensível e útil, } \\
\text { tendo a maioria dos doentes atingido os objetivos mínimos traçados inicialmente. Na perspectiva do doente, } \\
\text { a maioria teve uma noção de benefício subjetivo com a aplicação da TBA. }\end{array}$ \\
\hline $\begin{array}{l}\text { Barbosa, A. C., } \\
\text { et al. }\end{array}$ & 2020 & $\begin{array}{l}\text { Efeitos adversos sistêmicos raros relatados após aplicação de TBA nos membros inferiores incluem } \\
\text { fraqueza muscular distante do local da injeção, disfagia, boca seca, entre outros. }\end{array}$ \\
\hline Lauaté, J., et al. & 2004 & Este estudo confirma que a toxina botulínica pode melhorar o conforto de pacientes com espasticidade de \\
\hline
\end{tabular}




\begin{tabular}{|l|l|l|}
\hline & & $\begin{array}{l}\text { membros superiores e aumento da preensão palmar, além de melhorar e otimizar estratégias para o } \\
\text { tratamento da espasticidade focal dos membros inferiores. }\end{array}$ \\
\hline Slawek, J., et al. & 2005 & $\begin{array}{l}\text { Obtiveram um resultado estatisticamente significativo na redução do tônus muscular e do posicionamento } \\
\text { das mãos, na capacidade de realizar tarefas motoras simples e um efeito de curta duração de melhoria na } \\
\text { função do braço e funções simples da mão. Os movimentos muito complexos não diferiram } \\
\text { significativamente após aplicação da injeção, embora, em alguns casos individuais os pacientes foram } \\
\text { capazes de alcançar melhorias nesse aspecto. }\end{array}$ \\
\hline $\begin{array}{l}\text { Elovic, E. P., et } \\
\text { al. }\end{array}$ & 2008 & $\begin{array}{l}\text { Até 5 tratamentos com TBA entre 12 e 56 semanas em pacientes com espasticidade pós-AVE foi bem } \\
\text { tolerado e melhorou significativamente o tônus muscular, com deficiência diminuída e melhor qualidade de } \\
\text { vida dos pacientes. }\end{array}$ \\
\hline Kaji, R., et al. & 2010 & $\begin{array}{l}\text { TBA em altas doses reduziu a espasticidade nos músculos dos membros superiores e melhorou o } \\
\text { desempenho nas atividades de vida diária dos pacientes em termos de posição do membro e curativo. Além } \\
\text { disso, nenhuma diferença clinicamente relevante ou intercorrências foram observadas na frequência dos } \\
\text { pacientes das amostras. }\end{array}$ \\
\hline $\begin{array}{l}\text { Demetrios, M., } \\
\text { et al. }\end{array}$ & 2013 & $\begin{array}{l}\text { A injeção de TBA, seguida por terapia de movimento induzida por restrição modificada (mCIMT), mostrou } \\
\text { melhorar a espasticidade e a função motora do membro superior em pacientes com AVE crônico com } \\
\text { atividade motora residual voluntária do membro superior. }\end{array}$ \\
\hline
\end{tabular}

Fonte: Autores.

As neurotoxinas, de modo geral, atuam irrompendo a transmissão do impulso nervoso da célula neuronal, seu papel principal é afetar o processo de exocitose das vesículas sinápticas que produzem um bloqueio na liberação de acetilcolina, gerando assim, uma debilidade muscular prolongada (Barbosa, 2017). Seu uso para melhorar a assimetria da face em pacientes com paralisia facial adjacentes às sequelas do AVE, principalmente por meio da toxina botulínica, é um procedimento minimamente invasivo, muito eficiente e que apresenta alto índice de satisfação para os pacientes, e, por isso, vem sendo comumente utilizado, mesmo que ainda não exista um protocolo único de aplicação (Figallo et al., 2020).

Segundo Cardoso (2003), a Toxina Botulínica tipo A (TBA) é uma terapia farmacológica que traz efeitos benéficos ao paciente com espasticidade, como o ganho de amplitude de movimento e melhora da função dos membros afetados. O tratamento da espasticidade com a toxina botulínica tem maior êxito quando a administração segue alguns critérios, como: o ajuste da dose de acordo com a idade, com o peso, grau de espasticidade e musculatura administrada (Sposito, 2009). Aliado a isso, existem evidências de que a fisioterapia nesses pacientes se torna mais eficiente quando o tratamento é associado à TBA, especialmente quando essa intervenção ocorre durante o primeiro mês após a aplicação, haja vista que essa substância parece produzir melhoras na qualidade de vida, na função motora, espasticidade e amplitude de movimento (Oliveira et al., 2017).

No paciente com sequelas pós AVE, quando a espasticidade se torna incapacitante, a toxina botulínica do tipo A (BTX-A) é uma opção de tratamento frequentemente oferecida em combinação com terapia física e ocupacional. No membro superior, mecanicamente, a BTX-A pode reduzir o tônus adutor no ombro e o tônus flexor no cotovelo, punho e dedos. Embora os objetivos específicos do tratamento variem de acordo com as preferências do paciente e do cuidador, eles geralmente incluem função aprimorada, amplitude de movimento passiva e ativa aumentada, melhor higiene das mãos, redução da dor, capacidade aprimorada de controlar a posição do membro e maior facilidade de vestir (Foley, et al., 2013) Portanto, acredita-se que pacientes com sequelas de AVE apresentam melhora da qualidade de vida e capacidade funcional após aplicação da toxina botulínica tipo A (Gouvêa et al., 2015). Em estudo de Elovic et al. (2008), o tratamento com TBA por até 12 meses, aliado à fisioterapia, resultou em melhorias estatisticamente significativas nos pacientes com deficiência funcional decorrente das sequelas do AVE.

Desse modo, nota-se que a TBA representa a terapia padrão-ouro para espasticidade focal após acidente vascular cerebral, com baixa prevalência de complicações, reversibilidade e eficácia na redução da hipertonia espástica. (Souza et al., 2016). Portanto, está indicada e é reconhecida como o tratamento mais eficaz de duração temporária para devolver a simetria 
em pacientes com paralisia facial, porém é necessário conhecimento teórico e técnico de profissionais especializados para tratar e reabilitar pacientes com esse tipo de alteração. (Anjos et al., 2020), haja vista sua capacidade de contribui para melhorar não só a espasticidade em si, mas também para melhorar as dificuldades nas atividades de vida diária associadas à espasticidade de membros superiores (Fujimura, 2017).

Segundo Lima et al. (2020), os efeitos clínicos começam a aparecer a partir de 2 a 10 dias após a injeção, e o efeito máximo visível acorre após 14 dias após a aplicação, e o que determina o número de unidades aplicadas é a indicação e os tamanhos dos músculos envolvidos. Além disso, recomendações pós aplicação são necessárias, visto que, para ter um bom resultado, deve-se respeitar a não realização de qualquer exercício físico, viagens aéreas, abaixar a cabeça nas primeiras 4 horas. Além disso, o tratamento da espasticidade com a toxina botulínica tem maior êxito quando a administração segue alguns critérios, como: o ajuste da dose de acordo com a idade, com o peso, grau de espasticidade e musculatura administrada (Sposito, 2009).

Estudo de Wolfgang et al. (2014) e tiveram como resultado a diminuição dos padrões dolorosos, haja vista que, independentemente da ação da TB sobre o estado de tensão muscular, resultados também são encontrados na diminuição do limiar doloroso, pois a mesma pode atuar também no bloqueio de neurotransmissores liberados por nociceptores. Já no que se refere ao sistema nervoso central, Veverka et al. (2016) avaliou a ativação do córtex motor e do cerebelo, por meio de ressonância magnética. Segundo o autor, a intervenção da TB acompanhada da cinesioterapia é causadora do aumento da ativação de determinadas áreas do SNC, em decorrência do incremento das informações aferentes para o encéfalo.

O estudo de Serrano et al. (2014) constatou que o tratamento da espasticidade do membro superior com toxina botulínica do tipo A foi visivelmente bem tolerado e eficaz em termos de redução da hipertonia espástica e no atingimento de objetivos individualizados. Os resultados sugerem que o tratamento com TBA é seguro e eficaz na redução da espasticidade nas articulações avaliadas e na melhoria das amplitudes articulares do membro superior em complementaridade com o programa convencional de reabilitação. As associações realizadas entre as variáveis evidenciaram a existência de chances maiores para ocorrência do desfecho com uso da toxina botulínica tipo $\mathrm{A}$, em relação à espasticidade e funcionalidade quando comparado aos indivíduos que não receberam essa intervenção (Lauaté, et al., 2004). Segundo Pimentel, et al. (2014), o efeito benéfico da TBA na espasticidade dos membros superiores é mesmo notório, incluindo melhora na subescala motora da Medida de Independência Funcional (MIF). No entanto, o autor não conseguiu estabelecer a eficácia dessa substância no tratamento da espasticidade dos membros inferiores.

Dentre os efeitos colaterais, o uso da TBA pode provocar dor, astenia, hematomas ou equimose nos locais de aplicação. Raras vezes, podem acontecer reações sistêmicas, como síndrome gripal e fraqueza muscular em músculos distantes da injeção (Casaca, 2006). Aliado a isso, Barbosa et al (2020) alega que osefeitos adversos são geralmente de dois tipos: aqueles relacionados à injeção local; e aqueles relacionados aos efeitos sistêmicos da disseminação da toxina, e que fraqueza contralateral após o tratamento com TBA é um efeito adverso raramente relatado.

Não existe um consenso na literatura sobre a dosagem limite para o aparecimento dos efeitos indesejáveis da TBA. Casaca (2006) sugere que a dosagem de aplicação dessa toxina não deve exceder entre 600 a $800 \mathrm{U}$, enquanto que Portella et al. (2020) sugerem a dosagem limite de 300 a $400 \mathrm{U}$, reportando que pacientes que recebem doses anuais acima de $500 \mathrm{U}$ apresentam progressivamente mais chances de desenvolver anticorpo para a TBA. A melhor forma de uso parece ser, então, a aplicação em doses reduzidas, em áreas menores, e com um intervalo não muito longo entre as aplicações.

Resultados do estudo de Kaji et al. (2020) demonstraram que a dose de TBA entre 200 e 240 U tem eficácia clínica para o tratamento do pulso e dos dedos de pacientes com espasticidade pós AVE, sem eventos adversos limitantes de dose, o que pode ser vantajoso quando os efeitos colaterais são uma desvantagem na prática clínica. No entanto, não existe contraindicação absoluta para a utilização da TBA, porém, as contraindicações relativas são: precauções com a gravidez e 
lactação, com o uso de antibióticos, com doenças neuromusculares e com hipersensibilidade à toxina (Casaca, 2006). Desta forma, conclui-se que a toxina botulínica pode ser usada em associação com a fisioterapia no tratamento da espasticidade de maneira segura, desde que sejam obedecidas as recomendações do uso (Barbosa, et al., 2020).

Portanto, infere-se que a aplicação da TBA resulta na diminuição da espasticidade presente em indivíduos com lesão cerebral, e que esse medicamento pode ser aplicado utilizando-se técnicas diferentes para cada paciente, sendo que a duração dos efeitos é variada entre eles. Além disso, os efeitos observados em uma aplicação nem sempre são evidenciados em aplicações subsequentes em um mesmo indivíduo (Portella et al., 2004). Desse modo, a melhora da atividade funcional, espasticidade e deambulação, como relatado em estudo de Ward, et al. (2014), são capazes de retratar a diminuição das sequelas motoras pós AVE, alcançando, assim, uma das últimas fases do tratamento clínico: o restabelecimento funcional.

A administração deste fármaco está limitada a músculos específicos em doses controladas. Recomenda-se a injeção intramuscular em cada músculo comprometido com a espasticidade. Injeções subcutâneas podem ser indicadas em situações especiais. Assim, as injeções de TBA são um método valioso, reversível e seguro de tratamento, resultando em uma ampla gama de benefícios, com melhoria funcional mensurável. No entanto, a elaboração do plano terapêutico deve ser claramente estabelecida antes da terapia com a toxina, pois a melhora funcional é mais empírica naqueles pacientes com força muscular residual e função da mão, por isso, o tratamento deve ser individualizado em todos os casos (Slawek et al., 2005). Desse modo, a prática clínica para essa terapia deve ser baseada em um nível de julgamento profissional, por meio de estudos clínicos e experiencia empírica dos profissionais, e mais ensaios são necessários para facilitar a prática médica baseada em evidência (Demetrios et al., 2013)

\section{Conclusão}

Nota-se que o AVE continua sendo uma das grandes preocupações da atualidade, haja vista que é uma doença intimamente relacionada ao declínio da capacidade funcional e qualidade de vida, com quadro clínico é variado, e sequelas, na grande maioria dos casos, como a hemiparesia ou hemiplegia espástica. A toxina botulínica tipo A, que age através do bloqueio da transmissão neuromuscular via inibição da liberação de acetilcolina, é um tratamento bem estabelecido para espasticidade pós-AVE, com resultados clínicos favoráveis. No entanto, é de suma importância que se avalie de maneira singular cada paciente, bem como que a equipe multidisciplinar trace um plano terapêutico com base em suas limitações e funcionalidades preservadas.

Haja vista que a TBA bloqueia a hiperatividade muscular, produz o relaxamento da musculatura e atua na liberação de diversas substancias dos neurônios que promovem a espasticidade, bem como impede a liberação de substâncias nociceptivas, a aplicação dessa toxina, aliado à fisioterapia continuada, é o tratamento de primeira escolha para a espasticidade muscular, particularmente em pacientes que apresentam sequelas pós acidente vascular cerebral, com o intuito de aumentar a mobilidade, amplitude de movimento, facilitar a realização da higiene e de outras atividades funcionais, melhorar o desgaste da imobilização e a dor, e, dessa maneira, promover a melhoria da qualidade de vida desses pacientes. Dessa forma, estudos posteriores serão de suma relevância para melhor qualidade empírica deste tratamento, haja vista que se trata de uma propedêutica relativamente atual e ainda com poucas evidências afincas.

\section{Referências}

Almeida, S. R. M. (2012). Análise epidemiológica do acidente vascular no Brasil. Revista Neurociências, 20 (4), 481-482. https://doi.org/10.34024/rnc.2012.v20.8219

Anjos, A. A., Moura, B. A., Lima, J. M. C. S. R \& Lima, P. C. S. R. (2020). Toxina botulínica para correção de assimetria facial pós AVE: relato de caso clínico. Research, Society and Development, 9 (10), 1-17. https://doi.org/10.33448/rsd-v9i10.9214 
Barbosa, A. C., Ribeiro, I. M \& Medeiros, L. (2020). Paresia do membro superior contralateral após infiltração de toxina botulínica A para espasticidade pósAVE. Revista Científica da Ordem dos Médicos, 33 (11), 761-764. https://doi.org/10.20344/amp.11503

Barbosa, C. M. R. \& Barbosa, J. R. A. (2017). Toxina botulínica em Odontologia. Elsevier.

Calil, A. M. \& Paranhos, W. Y. (2007). O enfermeiro e as situações de emergência. Atheneu.

Cardoso, E., Pedreira, G., Prazeres, A., Nildo, R. \& Melo, A. (2007). Does Botulinum Toxin improve the function of the patient with spasticity after stroke? Arq Neuropsiquiatr, 63(3), 592-595. https://doi.org/10.1016/j.rehab.2018.05.1320

Casaca, I. (2006). Fisioterapia e Toxina Botulínica do Tipo A. Arq Fisioter, 1(2), 46-53.

Demetrios, M., Khan F., Stokes, L. T. M., Brand, C. \& McSweeney, S. (2013). Multidisciplinary rehabilitation following botulinum toxin and other focal intramuscular treatment for post-stroke spasticity. Cochrane Database of Systematic Reviews, 5 (6). https://doi.org/10.1002/14651858.CD009689

Elovic, E. P., Brashear, A., Kaelin D., Liu, J., Millis, R. S., Barron, R. \& Turkel, C. (2008). Repeated Treatments With Botulinum Toxin Type A Produce Sustained Decreases in the Limitations Associated With Focal Upper-Limb Poststroke Spasticity for Caregivers and Patients. Arch Phys Med Rehabil, 89 (1), 799-806. https://doi.org/10.1016/j.apmr.2008.01.007

Ênia, J. R. N., Fernandes, J. G. A, Nascimento, F., Silva, L. A. M., Reis, T. \& Dietrich, L. (2021). Toxina botulínica no tratamento da paralisia facial: um tratamento reabilitador minimamente invasivo. Research, Society and Development, 10 (5), 1-11. http://dx.doi.org/10.33448/rsd-v10i5.15204

Figallo, M. A. S., Hernández, G. R. L., Lagares, D. T., Araya, A. C., Ferrerosa, O. T., Pacheco, E. H. \& Perez, J. L. G. (2020). Use of Botulinum Toxin in Orofacial Clinical Practice. Toxins, 12(2), 1-16. https://doi.org/10.3390/toxins12020112

Foley, N., Pereira S., Salter, K., Fernandez, M. M., Speechley, M., Sequeira, K., Miller, T. \& Teasell, R. (2013). Treatment with botulinum toxin improves upper-extremity function post stroke: a systematic review and meta-analysis. Archives of Physical Medicine and Rehabilitation, 94 (5), 977-989. https://doi.org/10.1016/j.apmr.2012.12.006

Fujimura, O. T. R. K. (2017). Improvement in Disability Assessment Scale after Botulinum toxin A treatment for upper limb spasticity. Japanese Journal of Comprehensive Rehabilitation Science, 8 (1), 4-9. https://doi.org/10.11336/jjcrs.8.4

Gouvêa, D., Gomes, C. S. P., Melo, S. C., Abrahão, P. N. \& Barbieri, G. (2015). Acidente Vascular Encefálico: uma revisão da literatura. Ciência Atual: Revista Científica Multidisciplinar da Universidade São José, 6 (2), 2-6.

Kaji, R., Osako, Y., Suyama, K., Maeda, T., Uechi, Y. \& Iwasaki, M. (2010). Botulinum toxin type A in post-stroke upper limb spasticity. Current Medical Research \& Opinion, 36 (8), 1983-1992. https://doi.org/10.1185/03007995.2010.497103

Lauaté, J., Landrault, E., Courtois, S. J., Mertens, P. \& Boisson, D. G. (2004). Traitement de la spasticité focale du membre supérieur par toxine botulinique après accident vasculaire cérébral (AVE). Intérêt d'une approche au cas par cas. Annales de Réadaptation et de Médecine Physique, 47 (1), 555-562. https://doi.org/0.1016/j.annrmp.2004.02.01

Lima, L. S. R. \& Guedes, J. L. S. (2020). Botulinum toxin in dentistry: a literature. Review. Rev. Brá, 77 (1), 1-8. tp://dx.doi.org/10.18363/rbo.v77.2020.e1798

Lundstrom, E., Terént, A. \& Borg, L. (2008). Prevalence of disabling spasticity 1 year after first-ever stroke. European Journal of Neurology, 15 (6), 533-539. https://doi.org/10.1111/j.1468-1331.2008.02114.x

Mukai, Y. \& Kaji, R. (2011). Use of botulinum neurotoxin therapy. Brain and Nerve 63 (7), 775-784. https://doi.org/10.11477/mf.1416100959

Oliveira, D. R. N, Paiva, I. M. B. \& Anomal, R. F (2017). O uso da toxina botulínica no tratamento da espasticidade após acidente vascular encefálico: uma revisão de literatura. Journals Bahiana School of Medicine and Public Health, 7 (12), 289-297. http://dx.doi.org/10.17267/2238-2704rpf.v7i2.1242

Piassaroli, C. A. P, Almeida, G. C., Luvizotto, C. \& Suzan, A. B. B. M. (2012). Physical Therapy Rehabilitation Models in Adult Patients with Ischemic Stroke Sequel. Revista Neurociências, 20 (1), 128-137. https://doi.org/10.34024/rnc.2012.v20.10341

Pierson, S. H., Katz, D. L. \& Tarsy, D. (1996). Botulinum toxin A in the treatment of spasticity: functional implications and patient selection. Archives of Physical Medicine and Rehabilitation, 77 (7), 717-721. https://doi.org/10.1016 / s0003-9993 (96) 90015-5.

Pimentel, L. H. C., Alencar, F. J., Rodrigues, L. R. S., Sousa, F. C. F. \& Teles, J. B. M. (2014). Effects of botulinum toxin type A for spastic foot in poststroke patients enrolled in a rehabilitation program. Arq Neuropsiquiatr, 22 (1), 28-32. https://doi.org/10.1590/0004-282x20130189

Portella, L. V., Santiago, F. L. D., Maia, P. A. \& Mancini, M. C. (2004). Os efeitos da toxina botulínica no tratamento da espasticidade: uma revisão da literatura. Revista da Fisioterapia da Universidade de São Paulo, 11 (1), 47-55. https://doi.org/10.1590/fpusp.v11i1.76883

Schuster, R. C., Sant, C. R. \& Dalbosco, V. (2007). Efeitos da estimulação elétrica funcional (FES) sobre o padrão de marcha de um paciente hemiparético. Acta Fisiátrica, 14 (2), 82-86. https://doi.org/10.11606/issn.2317-0190.v14i2a102794

Serrano S., Constantino, J., Januário, F. \& Amaral, C. (2014). Espasticidade do Membro Superior: Avaliação da Eficácia e Segurança da Toxina Botulínica e Utilidade da Escala GAS - Estudo Retrospetivo. Rev Soc Port Med Fis Reabil, 25 (1), 11-19. http://dx.doi.org/10.25759/spmfr.127

Slawek J., Bogucki, U. \& Reclawowicz, D. (2005). Botulinum toxin type A for upper limb spasticity following stroke: an open-label study with individualised, flexible injection regimens. Neurol Sci, 26 (1), 32-39. https://doi.org/ 10.1007 / s10072-005-0379-8.

Souza, A. O. \& Cavalcanti, D. S. P. (2016). Toxina Botulínica Tipo A: aplicação e particularidades no tratamento da espasticidade, do estrabismo, do blefaroespasmo e de rugas faciais. Saúde \& Ciência em Ação, 3 (1), 58-70. 
Research, Society and Development, v. 10, n. 8, e56610817880, 2021

(CC BY 4.0) | ISSN 2525-3409 | DOI: http://dx.doi.org/10.33448/rsd-v10i8.17880

Sposito, M. M. M. (2004). Toxina botulínica tipo A: propriedades farmacológicas e uso clínico. Acta Fisiátrica, 11 (1), 7-44. https://doi.org/ 10.11606/issn.2317-0190.v11isupl.1a102495

Sposito, M. M. M. (2009). Botulinic Toxin Type A: action mechanism. Acta Fisiátrica, 16 (1), 25-37.

Teles, M. S. \& Gusmão, C. (2012). Fugl-Meyer Functional Evaluation of Patients patients with Stroke using the protocol of Fugl-Meyer. Revista Neurociências, 20 (1), 42-49.

Thompson, A. J., Jarrett, L., Lockley, J., Marsden, J. \& Stevenson VL (2005). Clinical Management of spasticity. Journal of Neurology, Neurosurgery, and Psychiatry, 76 (4), 459-463. https://doi.org/10.1136 / jnnp.2004.035972

Veverka, T., Hluštík, P., Hok, P., Otruba, P., Zapletalová, J., Zapletalová, J., Tüdös, Z., Alois, K. A. \& Kaňovský, P. (2016). Sensorimotor modulation by botulinum toxin A in post -stroke arm spasticity: Passive hand movement. J Neurol Sci, Czech Republic, 362 (3), 14-20.

Ward, A. B., Wissel, J., Borg, J., Ertzgaard, P., Herrmann, C., Kulkarni, J., Lindgren, K., Reuter, I., Sakel, M., Satero, P., Sharma, S., Wein, T., Wright, N. \& Smith, A. F. (2014). Functional goal achievement in post-stroke spasticity patients: the BOTOX Economic Spasticity Trial (BEST). J Rehabil Med. 46 (6), 504-513. https://doi.org/ 10.2340 / 16501977-1817

Wolfgang, J. H., Hefter, H., Reissig, A., Kollewe, K. \& Wissel, J. (2014). Efficacy and safety of botulinum toxin type A (Dysport) for the treatment of poststroke armspasticity: Results of the German-Austrian open-label post-marketing surveillance prospective study. J Neurol Sci, 337 (1), $86-90$. https://doi.org/10.1016 / j.jns.2013.11.022 